\title{
Transfer of reactive inhibition as a function of prior training on the inhibitory task
}

\author{
SHANG-HWA HSU and R. B. PAYNE \\ University of Georgia, Athens, Georgia 30602
}

\begin{abstract}
Task-specific and task-nonspecific (Hullian) conceptions of reactive inhibition (IR) were examined in a dual-task paradigm in which two segments of massed practice on a main task were separated either by rest or by massed practice on an alternate task upon which subjects had received varying amounts of practice $24 \mathrm{~h}$ earlier. Given that prior practice on the alternate task increased the task specificity of its variance, and given reminiscence on the main task as an index of $I_{R}$ transfer from the alternate task, the task-specific conception of $I_{R}$ implies increasing main-task reminiscence as a function of the amount of prior training on the alternate task, whereas the task-nonspecific conception implies equal amounts of main-task reminiscence for all levels of prior training on the alternate task. Results from a sample of 120 subjects (60 of each sex) showed significant $I_{R}$ transfer for both sexes, and the amount transferred was independent of the amount of prior training. Thus, the task-nonspecific conception of $I_{R}$ was upheld.
\end{abstract}

In order to develop evidence bearing upon Hull's (1943) conception of reactive inhibition $\left(I_{R}\right)$ as a general negative motivational factor, several studies employed a dual-task paradigm in which subjects either rested or performed an alternate task between two segments of massed practice on a principal task. Given the capability of each task alone to generate evidence for $I_{R}$, and barring habit transfer effects from one task to the other, the rationale of this procedure was that the $I_{R}$ generated by the interpolated task should transfer to depress performance on the main task and, therefore, also to reduce main-task reminiscence relative to the effect of rest. Within this context, some of the studies found strong support for the Hullian position (Adams, 1955; Coppage, 1979; Duncan, 1957; Hsu \& Payne, 1979; Huang \& Payne, 1977) and others did not (Humphries \& McIntyre, 1963a, 1963b; McIntyre, Mostoway, Stojak, \& Humphries, 1972; Pavlik \& Schwartz, 1963). Noting that the alignments of mean scores on the critical trials were as one would expect under Hullian theory, Huang and Payne (1977) questioned the dependability of the results of McIntyre et al. (1972) on grounds of sample size, and Hicks (1972) offered a similar objection to the studies of Humphries and McIntyre (1963a, 1963b).

One outgrowth of the negative findings was the idea that $I_{R}$, as a conceptual tool for dealing with the relatively decremental effects of massed practice, should be regarded as task specific and therefore without reductive consequences for performance on other tasks (McIntyre et al., 1972). Although this modification of the $I_{R}$ concept departed radically from Hull's (1943) view, it nonetheless allowed the notion of work-produced inhibition to remain viable as an explanation of workrest phenomena within tasks. However, within the context of the dual-task paradigm, this modification implied unwittingly that the transferability of $I_{R}$ from the alternate task to the main task should be an increasing function of the similarity of the two tasks. This would mean that the $I_{R}$ generated in a highly similar alternate task would merely add to the pool of $I_{R}$ developed in the main task, whereas the $I_{R}$ produced by performance on a dissimilar alternate task would somehow remain isolated from this pool so as not to affect the recovery process on the main task. To the contrary, however, Huang and Payne (1977) found that work inhibition transferred equally from two alternate tasks to the main task, despite differences in their similarities to the main task.

Although the weight of evidence seems to favor the Hullian variant of inhibition theory, studies of intertrial correlation patterns and their factorial content (Fleishman \& Hempel, 1954; Jones, 1962, 1966; Noble, 1970; Perl, 1934; Reynolds, 1952), as well as trial-totrial analyses of performance (Ammons, Ammons, \& Morgan, 1958; Archer, 1958), suggested that there may be special conditions under which the task-specificity conception of work inhibition is valid. Collectively, these studies seem to have shown that, as practice proceeds, irrelevant responses drop out, variance common to other tasks declines, and residual response patterns become more highly task specific. Such evidence raises the possibility that the results of Coppage (1979), Hsu and Payne (1979), and Huang and Payne (1977) favored the Hullian version of inhibition theory because their interpolated tasks were not learned well enough to ensure a high degree of task-specific variance and, therefore, also a high degree of task-specific inhibition.

Accepting the generalization that practice promotes a progressive task specificity of response patterns, it follows jointly from this principle and from the similarity 
implication of the task-specificity conception of $I_{R}$, noted earlier, that the amount of inhibition transferred from the interpolated task to the main task should vary inversely with the amount of prior practice on the interpolated task. By the same token, it also follows that the amount of main-task reminiscence across the interpolation interval should vary directly with the amount of prior practice on the interpolated task. When rest is employed as a control condition to assess the effect of interpolated-task practice on main-task reminiscence (hereafter called the interval effect), one should find a significant interaction between the interval effect and the prior-practice effect (hereafter called the learning effect). In other words, the effect of rest upon maintask reminiscence should be the same for all levels of prior practice on the interpolated task, whereas the reductive effect of the interpolated task on main-task reminiscence should decline as a function of the amount of prior practice. On the other hand, if Hull's (1943) characterization of $I_{R}$ as negative drive is valid, one should find that the depressant effect of interpolatedtask practice on main-task reminiscence is independent of the amount of prior practice. The present experiment was conducted in order to assess the relative merits of these contending views.

\section{METHOD}

\section{Main Experiment}

Subjects. The experimental sample consisted of 120 subjects (60 of each sex) drawn by consent from introductory psychology classes. All were Caucasian, right-handed, and normally sighted. Modal age was 18 years.

Tasks. The main task, mirror tracking, required subjects to use mirror vision in order to track a small silver target as it moved clockwise at $1 \mathrm{rpm}$ through a narrow star-shaped pathway. Subjects were instructed to keep the stylus on target as long as possible and to avoid contact with the sides of the pathway. Time on target was recorded in units of $.01 \mathrm{sec}$ for each $1 \mathrm{~min}$ of practice.

The interpolated task was provided by the USAF SAM Rotary Pursuit Test, Model CM803B2, as described by Melton (1947). Subjects were instructed to keep the stylus in contact with the target as it rotated clockwise at $60 \mathrm{rpm}$.

Procedure. Each subject participated in two experimental sessions that were separated by $24 \mathrm{~h}$. On Day 1 , the subject observed a brief demonstration of rotary pursuit performance and then practiced on rotary pursuit for 0,10 , or $2030 \mathrm{sec}$ trials, with intertrial intervals of $20 \mathrm{sec}$ (the learning treatment). These amounts of practice were selected on evidence that they would provide substantial variation in the amount of taskspecific variance (Fleishman, 1960). On Day 2, the operational sequence consisted of $5 \mathrm{~min}$ of massed practice on mirror tracking (all subjects), $5 \mathrm{~min}$ of rest or $5 \mathrm{~min}$ of massed practice on rotary pursuit (the interval treatment), and $10 \mathrm{~min}$ of massed practice on mirror tracking (all subjects). Thus, the independent variables of interest were sex of subject, amount of rotary pursuit practice on Day $1(0,10$, or 20 trials), and the nature of the interpolated activity on Day 2 (rest or rotary pursuit practice). The 60 subjects of each sex were assigned without bias to the six combinations of learning treatment and interval treatment except for the constraint that the combinations contain equal numbers of subjects.

Two special control problems require comment. First, a potential effect of postural shift on main-task reminiscence as the subject moved from the mirror-tracking station to the rotary pursuit station and back again was controlled by having the members of the rest group shift in the same manner. The rest-group subjects remained standing at the rotary pursuit station for all but the last $5 \mathrm{sec}$ of the rest period and then returned to the mirror-tracking station for resumption of practice. Second, we recognized the possibility of a confound between habit transfer effects and inhibition transfer effects for those subjects who received rotary pursuit as the interval treatment. However, faced with the same problem, Huang and Payne (1977) found no evidence of habit transfer from rotary pursuit to mirror tracking or vice versa. Thus, control groups addressed to this problem were omitted from the present study.

\section{Ancillary Experiment}

The foregoing experimental operations were considered sufficient to resolve the theoretical question at hand provided that the interaction between the learning effect and the interval effect turned out to be negligible, as predicted by Hullian theory. However, a significant interaction in which main-task reminiscence increased as a function of prior practice on the alternate task would not necessarily support the task-specific conception of $I_{R}$, because one would not know whether the interaction was due to (1) declining inhibition transfer as a function of increasing task specificity of response patterns resulting from prior training, as the theory predicts, or (2) reduction in the amount of inhibition generated and available for transfer as a function of prior training. The point is of some consequence because Ammons (1947) and Irion (1949) found that reminiscence at first increased and then decreased as a function of the amount of prerest practice. Although our choice of prior-practice levels was guided by the Ammons and the Irion data, as well as by the necessity to provide enough trials to ensure substantial variations in task-specific variance (Fleishman, 1960), it seemed imperative nonetheless to conduct an ancillary study in order to determine whether one could validly infer the generation of equal amounts of inhibition by the interpolated task for all levels of prior practice on it. Accordingly, 30 subjects (12 males, 18 females) were given 0,10 , or 20 rotary pursuit trials on Day 1, as in the main experiment. On Day 2, all subjects were exposed to an operational sequence consisting of $5 \mathrm{~min}$ of massed practice on mirror tracking, 5 min of massed practice on rotary pursuit, $5 \mathrm{~min}$ of rest, and two postrest trials on rotary pursuit. Reminiscence measured across the rest period was used as an index of the amount of inhibition generated by rotary pursuit and available for transfer.

\section{RESULTS}

\section{Ancillary Experiment}

A reminiscence score on rotary pursuit was computed for each subject by subtracting the performance score on the last prerest trial from that on the first postrest trial. A variance analysis of the 30 scores showed that mean reminiscence values were essentially the same for all levels of prior practice $[\mathrm{F}(2,24)<1]$. Thus, one can infer that the amount of inhibition generated and available for transfer was the same for all levels of prior practice.

\section{Main Experiment}

Experimental treatment effects were measured in terms of the amount of reminiscence across the interval between the two segments of practice on mirror tracking. For this purpose, a reminiscence score was computed for each subject by subtracting the performance score on Trial 5 from that on Trial 6. The 120 scores were then subjected to a variance analysis in which sex, 


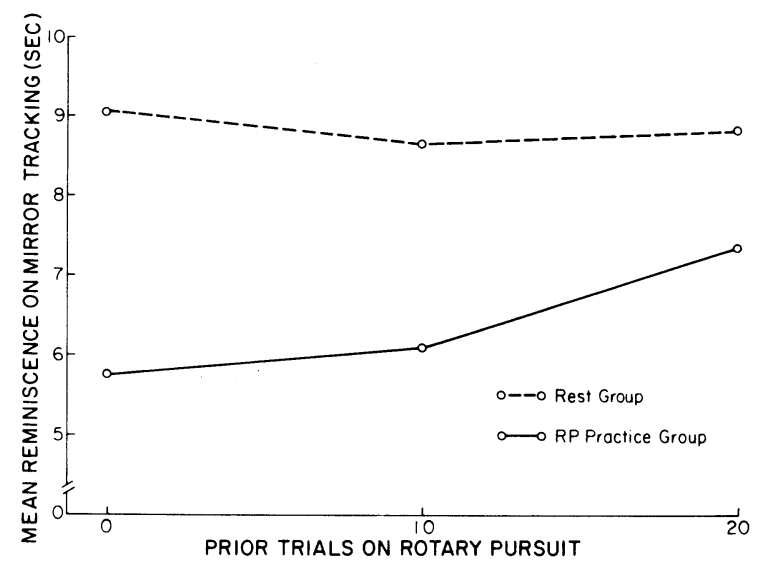

Figure 1. Main-task reminiscence as a joint function of learning and interval treatments.

interval treatment, and learning treatment composed the main effects.

With regard to the sex effect, females reminisced more than did males $[\mathrm{F}(1,108)=19.38, \mathrm{p}<.001]$ as many have found (e.g., Huang \& Payne, 1975; McBride $\&$ Payne, 1979). With regard to the interval effect, the results confirmed those of Coppage (1979) and Hsu and Payne (1979) by showing that the interpolation of rotary pursuit practice diminished mean reminiscence on mirror tracking relative to the effect of rest $[F(1,108)=$ $9.22, \mathrm{p}<.005]$. No other effects were significant (p>.05).

The lack of significant evidence for an interaction between the learning effect and the interval effect $[\mathrm{F}(2,108)<1]$ is decisive for the theoretical alternatives under study because it implies that the inhibitory potential generated by interpolated rotary pursuit practice transferred to suppress reminiscence on mirror tracking without regard for the amount of prior practice. This outcome can be visualized more concretely by reference to Figure 1 , in which mean reminiscence is plotted as a joint function of prior learning and the conditions that prevailed during the interpolation interval. Although there is a slight suggestion that the two curves may not be parallel, analysis showed that the interaction variance remained nonsignificant even when all of it was assigned to the linear component of the learning effect $[F(1,108)=1.87, p>.1]$. Statistically, therefore, the two curves can be regarded as parallel, and one can conclude that variations in the amount of prior training on the inhibitory task affected neither the amount of inhibition available for transfer nor the amount actually transferred.

\section{DISCUSSION}

The empirical fact of reminiscence within tasks can be deduced from either the task-specific or the task-nonspecific conception of reactive inhibition, but the results of the present study can be deduced only from the latter conception. What makes this deduction possible, of course, is Hull's (1943) assump- tion that $I_{R}$ has primary negative motivational properties, from which it follows that practice on an interpolated task should reduce reminiscence on the main task. The present results provided unequivocal support for this assumption.

The results also can be regarded as decisive evidence against arousal and consolidation theories of reminiscence. According to arousal theory (Catalano, 1967; Catalano \& Whalen, 1967), as amplified by McIntyre et al. (1972), massed practice produces an arousal decrement that is reflected in a relatively diminished level of performance. Various events, such as rest, task change, induced muscular tension, and the like, are presumed to reverse the arousal decrement and produce the gains in performance observed as reminiscence. On this hypothesis, reminiscence on mirror tracking in the interpolated-task group should have been at least equal to that shown by the rest group, but it was not. The results, therefore, negated a clear prediction based on arousal theory, as previous results have done (Coppage, 1979; Hsu \& Payne, 1979).

Consolidation theory assumes that neural processes involved in learning remain active for a while after practice has ceased. This perseverative activity serves to consolidate and strengthen the associations formed in practice. Introducing a subsequent trial too soon interferes with consolidation and results in relatively slow progress. By contrast, a rest period facilitates consolidation, the effect of which is observed as reminiscence. Applying the theory to reminiscence in rotary pursuit learning, Eysenck and Frith (1977) assumed further that the highly specific variance of rotary pursuit performance rendered other tasks incapable of interrupting its consolidation process, perhaps because other tasks would not engage precisely the same area of the central nervous system. By the same token, of course, rotary pursuit practice should not interfere with the consolidation processes of other tasks. On this theory, consolidation in the main task of the present study should have proceeded without interruption under the interpolated-task condition, and reminiscence should have occurred as though the treatment interval had been filled with rest. Needless to repeat, this expectation was not realized, nor was it evident in the data of Coppage (1979) and Hsu and Payne (1979). The present study, therefore, contradicted a clear prediction based on consolidation theory.

\section{REFERENCES}

Adams, J. A. A source of decrement in psychomotor performance. Journal of Experimental Psychology, 1955, 49, 390-394.

Ammons, R. B. Acquisition of motor skill: II. Rotary pursuit performance with continuous practice before and after a single rest. Journal of Experimental Psychology, 1947, 37, 393-411.

Ammons, R. B., Ammons, C. H., \& Morgan, R. L. Subskills in rotary pursuit as affected by rate and accuracy requirements and by distribution of practice. Journal of General Psychology, $1958,58,259-279$.

Archer, E. J. Effects of distribution of practice on a component skill of rotary pursuit tracking. Journal of Experimental Psychology, 1958, 56, 427-436.

Catalano, J. F. Arousal as a factor in reminiscence. Perceptual and Motor Skills, 1967, 24, 1171-1180.

Catalano, J. F., \& Whalen, P. M. Factors in recovery from performance decrement: Activation, inhibition, and warm-up. Perceptual and Motor Skills, 1967, 24, 1223-1231.

Coppage, S. J. An experimental test of theoretical conceptions of psychomotor reminiscence. Unpublished doctoral dissertation, University of Georgia, 1979.

Duncan, C. P. Visual and kinesthetic components of reactive inhibition. American Journal of Psychology, 1957, 70, 616-619.

Eysenck, H. J., \& Frith, C. D. Reminiscence, motivation, and personality. New York: Plenum, 1977.

Fle ishman, E. A. Abilities at different stages of practice in rotary pursuit performance. Journal of Experimental Psychology, 1960, 60, 162-171. 
Fleishman, E. A., \& Hempel, W. E. Changes in factor structure of a complex psychomotor test as a function of practice. Psychometrika, 1954, 19, 239-252.

Hicks, R. E. Bilateral reminiscence in inverted-reversed printing Effects of interpolated activity and printing-hand sequence. Journal of Motor Behavior, 1972, 4, 241-250.

Hsu, S. H., \& PAYNe, R. B. Effector localization and transfer of reactive inhibition. Journal of Motor Behavior, 1979, 11, 153 158.

Huang, K. L., \& Payne, R. B. Individual and sex differences in reminiscence. Memory \& Cognition, 1975, 3, 252-256.

Huang, K. L., \& PAyne, R. B. Transfer of reactive inhibition. Journal of Motor Behavior, 1977, 9, 293-300.

Hull, C. L. Principles of behavior. New York: AppletonCentury-Crofts, 1943.

Humphries, M., \& McIntyre, J. An attempt to find a locus of temporary work decrement in pursuit rotor performance. Perceptual and Motor Skills, 1963, 17, 397-398. (a)

Humphries, M., \& McInTYRe, J. Effect of interpolated monocular and binocular visual pursuit reaction time activity on reminiscence in pursuit rotor performance. Perceptual and Motor Skills, 1963, 17, 333-334. (b)

IrION, A. L. Reminiscence in pursuit-rotor learning as a function of length of rest and of amount of pre-rest practice. Journal of Experimental Psychology, 1949, 39, 492-499.

Jones, M. B. Practice as a process of simplification. Psychological Review, 1962, 69, 274-294.
JoNes, M. B. Individual differences. In E. A. Bilodeau (Ed.), Acquisition of skill. New York: Academic Press, 1966.

McBride, D. K., \& PAYNe, R. B. Psychomotor reminiscence as a function of sex and length of rest period. Journal of Motor Behavior, 1979, 11, 59-64.

McIntyre, J. S., Mostoway, W., Stojak, R. A., \& Humphries, M. Transfer of work decrement in motor learning. Journal of Motor Behavior, 1972, 4, 223-229.

Melton, A. W. (Ed.). Apparatus tests (AAF Aviation Psychology Program, Research Rep. 4). Washington, D.C: U.S. Government Printing Office, 1947.

Noble, C. E. Acquisition of pursuit tracking skill under extended training as a joint function of sex and initial ability. Journal of Experimental Psychology, 1970, 86, 360-373.

Pavlik, W. B., \& Schwartz, M. M. A test of reactive inhibition as drive. Perceptual and Motor Skills, 1963, 16, 101-103.

PERL, R. E. An application of Thurstone's method of factor analysis to practice series. Journal of General Psychology, 1934, 11, 209-212.

REYNolds, B. The effects of learning on the predictability of psychomotor performance. Journal of Experimental Psychology, $1952,44,189-198$

(Received for publication January 20, 1981.) 